\title{
Further Results about Traveling Wave Exact Solutions of the (2+1)-Dimensional Modified KdV Equation
}

\author{
Yang Yang $\mathbb{D},{ }^{1}$ Jian-ming Qi $\mathbb{D},,^{2}$ Xue-hua Tang $\mathbb{D},{ }^{3}$ and Yong-yi Gu $\mathbb{D}^{4}$ \\ ${ }^{1}$ Mechanics Institute, Shanghai Dianji University, Shanghai 201306, China \\ ${ }^{2}$ School of Business, Shanghai Dianji University, Shanghai 201306, China \\ ${ }^{3}$ School of Design and Art, Shanghai Dianji University, Shanghai 201306, China \\ ${ }^{4}$ School of Statistics and Mathematics, Guangdong University of Finance and Economics, Guangzhou 510320, China
}

Correspondence should be addressed to Jian-ming Qi; qijianmingdaxia@163.com and Yong-yi Gu; gdguyongyi@163.com

Received 27 February 2019; Revised 15 April 2019; Accepted 24 April 2019; Published 16 May 2019

Academic Editor: Qin Zhou

Copyright (C) 2019 Yang Yang et al. This is an open access article distributed under the Creative Commons Attribution License, which permits unrestricted use, distribution, and reproduction in any medium, provided the original work is properly cited.

We used the complex method and the $\exp (-\phi(z))$-expansion method to find exact solutions of the $(2+1)$-dimensional mKdV equation. Through the maple software, we acquire some exact solutions. We have faith in that this method exhibited in this paper can be used to solve some nonlinear evolution equations in mathematical physics. Finally, we show some simulated pictures plotted by the maple software to illustrate our results.

\section{Introduction}

Nonlinear science is basic science to study the generality of nonlinear phenomena. It is a comprehensive discipline which has been gradually developed by various branch disciplines characterized by nonlinearity since the 1960s [1]. It was known as the "Third Revolution" of Natural Science in the 20th century. The scientific community believes that the research of nonlinear science has not only great scientific significance but also broad application prospects. It involves almost all fields of natural science and social science, including engineering application, basic physical research, biological research, control theory, and management $[2,3]$. And the nonlinear science is changing people's traditional view of the real world. It is more and more important to find the exact solution of the nonlinear evolution equation. Therefore, a variety of solutions have emerged.

In 1967, Gardner and others [4] first proposed scattering inversion method for KdV equation. Since then, many methods and techniques for constructing nonlinear partial differential equations have been gradually proposed for seeking soliton solutions [5-7], such as Bäcklund transform [8], Hirota method [9-11], and Darboux transform [12]. But these methods are complex and difficult to use in solving processes. With the deepening of research and the continuous development of mathematical computing software, in recent years, some analytical tools and direct algebraic methods have gradually emerged, such as fixed point theorems [1316], variational methods [17-20], topological degree method [21-24], homogeneous balance method [25], tanh function method [26, 27], Jacobi elliptic function method [28, 29], F-expansion method [30, 31], and $\exp (-\phi(z))$-expansion method [32-34].

In 2012, Alejo [35] got some numerical results which showed a new family of solutions of the geometric $\mathrm{mKdV}$ equation. In 2014, Huang Y, Wu Y, and Meng F, et al. [36] used the complex method to get the meromorphic solutions of complex combined KdV-mKdV equation. Singh [37] used the Jacobian elliptic function expansion method to get the exact solutions of Wick-type stochastic KerstenKrasil' shchik coupled KdV-mKdV equations.

Consider the following:

$$
u_{t}+\tau u^{2} u_{x}+\beta u_{x x x}=0 .
$$

In (1)(see [38]), $\tau$ and $\beta$ are constants. 
Submitting $u(x, t)=w(z), z=k x+w t$, into (1) and after integrating, we get

$$
\beta k^{3} w^{\prime \prime}+\frac{k \tau}{3} w^{3}-\omega w+d=0
$$

In order to get the exact solution of $\mathrm{mKdV}$ equation, we use the complex method which was suggested by Yuan et al.
[39-42] to get solutions of (2), and then we also get some exact solutions by the $\exp (-\phi(z))$-expansion method.

Theorem 1. By using the complex method, we suppose that $\beta k^{4} \tau / 3 \neq 0$ and then all meromorphic solutions $w$ belong to the class $W$. And we found that there will be three forms of solutions of (2):

(1) The Elliptic Function Solutions

$$
w_{d}(z)= \pm \frac{1}{2} \sqrt{-\frac{\beta k^{2}}{\tau}} \frac{(-\wp+c)\left(4 \wp c^{2}+4 \wp^{2} c+2 \wp^{\prime} a-\wp g_{2}-c g_{2}\right)}{\left(\left(12 c^{2}-g_{2}\right) \wp+4 c^{3}-3 c g_{2}\right) \wp^{\prime}+\left(4 \wp^{3}+12 c \wp^{2}-3 g_{2} \wp-c g_{2}\right) d^{\prime}},
$$

in (3), $g_{3}=0, a^{2}=4 c^{3}-g_{2} c$, and $g_{2}$ and $c$ are arbitrary constants.

\section{(2) The Simply Periodic Solutions}

$$
\begin{array}{r}
w_{s, 1}(z)=\alpha \sqrt{-\frac{6 \beta k^{2}}{\tau}}\left(\operatorname{coth} \frac{\alpha}{2}\left(z-z_{0}\right)\right. \\
\left.-\operatorname{coth} \frac{\alpha}{2}\left(z-z_{0}-z_{1}\right)-\operatorname{coth} \frac{\alpha}{2} z_{1}\right),
\end{array}
$$

in (4), $z_{0} \in \mathbb{C}, \omega=-\beta k^{3} \alpha^{2}\left(1 / 2+3 / 2 \sinh ^{2}(\alpha / 2) z_{1}\right), d=$ $\sqrt{-6 \beta k^{2} / \tau} \tanh (\alpha / 2) z_{1} / \sinh ^{2}(\alpha / 2) z_{1}$, and $z_{1} \neq 0$.

And the other solution is

$$
w_{s, 2}(z)=\alpha \sqrt{-\frac{6 \beta k^{2}}{\tau}} \tanh \frac{\alpha}{2}\left(z-z_{0}\right)
$$

in (5), $z_{0} \in \mathbb{C}, \omega=-\beta k^{3} \alpha^{2} / 2, d=0$.

(3) The Rational Function Solutions

$$
w_{r, 1}(z)= \pm \sqrt{-\frac{6 \beta k^{2}}{\tau}} \frac{1}{z-z_{0}}
$$

and

$$
w_{r, 2}(z)= \pm \sqrt{-\frac{6 \beta k^{2}}{\tau z_{1}^{2}}}\left(\frac{z_{1}}{z-z_{0}}-\frac{z_{1}}{z-z_{0}-z_{1}}-1\right)
$$

in (6), $z_{0} \in \mathbb{C}, \omega=0, d=0$, or in (7), $z_{0} \in \mathbb{C}, \omega=-6 \beta k^{3} /$ $z_{1}^{2}, \quad d=\mp(2 / 3) k \tau\left(-6 \beta k^{2} / \tau z_{1}^{2}\right)^{3 / 2}$.

Theorem 2. By using $\exp (-\phi(z))$-expansion method, there will be three forms of solutions of (2).

$$
\begin{aligned}
& \text { If } \delta^{2}-4 \mu>0, \mu \neq 0, \\
& u_{11}(z)=\frac{1}{2} \lambda \sqrt{\frac{-6 \beta k^{2}}{\tau}+\sqrt{\frac{-6 \beta k^{2}}{\tau}}} \\
& \cdot \frac{2 \mu}{\sqrt{\left(\delta^{2}-4 \mu\right)} \tanh \left(\left(\sqrt{\delta^{2}-4 \mu} / 2\right)(z+c)+\delta\right)},
\end{aligned}
$$

$$
u_{12}(z)=\frac{1}{2} \lambda \sqrt{\frac{-6 \beta k^{2}}{\tau}}+\sqrt{\frac{-6 \beta k^{2}}{\tau}}
$$$$
\frac{2 \mu}{\sqrt{\left(\delta^{2}-4 \mu\right)} \operatorname{coth}\left(\left(\sqrt{\delta^{2}-4 \mu} / 2\right)(z+c)+\delta\right)}
$$$$
u_{21}(z)=-\frac{1}{2} \lambda \sqrt{\frac{-6 \beta k^{2}}{\tau}}-\sqrt{\frac{-6 \beta k^{2}}{\tau}}
$$$$
\frac{2 \mu}{\sqrt{\left(\delta^{2}-4 \mu\right)} \tanh \left(\left(\sqrt{\delta^{2}-4 \mu} / 2\right)(z+c)+\delta\right)}
$$$$
u_{22}(z)=-\frac{1}{2} \lambda \sqrt{\frac{-6 \beta k^{2}}{\tau}}-\sqrt{\frac{-6 \beta k^{2}}{\tau}}
$$$$
\frac{2 \mu}{\sqrt{\left(\delta^{2}-4 \mu\right)} \operatorname{coth}\left(\left(\sqrt{\delta^{2}-4 \mu} / 2\right)(z+c)+\delta\right)} \text {. }
$$

$$
\text { If } \delta^{2}-4 \mu<0, \mu \neq 0 \text {, }
$$$$
u_{13}(z)=\frac{1}{2} \lambda \sqrt{\frac{-6 \beta k^{2}}{\tau}}+\sqrt{\frac{-6 \beta k^{2}}{\tau}}
$$

$$
\frac{2 \mu}{\sqrt{\left(\delta^{2}-4 \mu\right)} \tanh \left(\left(\sqrt{\delta^{2}-4 \mu} / 2\right)(z+c)+\delta\right)}
$$

$$
u_{14}(z)=\frac{1}{2} \lambda \sqrt{\frac{-6 \beta k^{2}}{\tau}}+\sqrt{\frac{-6 \beta k^{2}}{\tau}}
$$

$$
\frac{2 \mu}{\sqrt{\left(\delta^{2}-4 \mu\right)} \operatorname{coth}\left(\left(\sqrt{\delta^{2}-4 \mu} / 2\right)(z+c)+\delta\right)},
$$

$$
\begin{gathered}
u_{23}(z)=-\frac{1}{2} \lambda \sqrt{\frac{-6 \beta k^{2}}{\tau}}-\sqrt{\frac{-6 \beta k^{2}}{\tau}} \\
\cdot \frac{2 \mu}{\sqrt{\left(\delta^{2}-4 \mu\right)} \tanh \left(\left(\sqrt{\delta^{2}-4 \mu} / 2\right)(z+c)+\delta\right)},
\end{gathered}
$$




$$
\begin{aligned}
& u_{24}(z)=-\frac{1}{2} \lambda \sqrt{\frac{-6 \beta k^{2}}{\tau}}-\sqrt{\frac{-6 \beta k^{2}}{\tau}} \\
& \cdot \frac{2 \mu}{\sqrt{\left(\delta^{2}-4 \mu\right)} \operatorname{coth}\left(\left(\sqrt{\delta^{2}-4 \mu} / 2\right)(z+c)+\delta\right)} \text {. } \\
& \text { If } \delta^{2}-4 \mu>0, \mu=0, \delta \neq 0, \\
& u_{15}(z)=\frac{1}{2} \lambda \sqrt{\frac{-6 \beta k^{2}}{\tau}}+\sqrt{\frac{-6 \beta k^{2}}{\tau}} \frac{\delta}{\exp (\delta(z+c))-1}, \\
& u_{25}(z)=-\frac{1}{2} \lambda \sqrt{\frac{-6 \beta k^{2}}{\tau}} \\
& -\sqrt{\frac{-6 \beta k^{2}}{\tau}} \frac{\delta}{\exp (\delta(z+c))-1} \\
& \text { If } \delta^{2}-4 \mu=0, \mu \neq 0, \delta \neq 0, \\
& u_{16}(z)=\frac{1}{2} \lambda \sqrt{\frac{-6 \beta k^{2}}{\tau}}+\sqrt{\frac{-6 \beta k^{2}}{\tau}} \frac{\delta^{2}(z+c)}{2(\delta(z+c)+2)}, \\
& u_{26}(z)=-\frac{1}{2} \lambda \sqrt{\frac{-6 \beta k^{2}}{\tau}}-\sqrt{\frac{-6 \beta k^{2}}{\tau}} \frac{\delta^{2}(z+c)}{2(\delta(z+c)+2)} . \\
& \text { If } \delta^{2}-4 \mu=0, \mu=0, \delta=0 \text {, } \\
& u_{17}(z)=\frac{1}{2} \lambda \sqrt{\frac{-6 \beta k^{2}}{\tau}}+\sqrt{\frac{-6 \beta k^{2}}{\tau}} \frac{1}{z+c} \\
& u_{27}(z)=-\frac{1}{2} \lambda \sqrt{\frac{-6 \beta k^{2}}{\tau}}-\sqrt{\frac{-6 \beta k^{2}}{\tau}} \frac{1}{z+c} \text {. }
\end{aligned}
$$

\section{Preliminary Lemmas, Complex Method and $\exp (-\phi(z))$-Expansion Method}

2.1. Introduction of Complex Method. For the introduction of complex method, we have to know some concepts and symbols.

Lemma 3 (see [34]). First we set $m \in \mathbb{N}:=\{1,2,3 \ldots\}, r_{j} \in$ $\mathbb{N}_{0}=\mathbb{N} \cup\{0\}, r=\left(r_{0}, r_{1}, \ldots, r_{m}\right), j=0,1, \ldots, m$. Then we can get a differential monomial by

$$
\begin{aligned}
M_{r} & {[w](z) } \\
& :=[w(z)]^{r_{0}}\left[w^{\prime}(z)\right]^{r_{1}}\left[w^{\prime \prime}(z)\right]^{r_{2}} \cdots\left[w^{(m)}(z)\right]^{r_{m}} .
\end{aligned}
$$

$p(r):=r_{0}+2 r_{1}+\cdots+(m+1) r_{m}$ and $\operatorname{deg}(M)$ are regarded as the weight and degree of $M_{r}[w]$, separately.

The differential polynomial $P\left(w, w^{\prime}, \ldots, w^{(m)}\right)$ can be defined as follows:

$$
P\left(w, w^{\prime}, \ldots, w^{(m)}\right):=\sum_{r \in I} a_{r} M_{r}[w]
$$

In (23), $a_{r}$ are constants, and I is a finite index set.

The total weight and degree of $P\left(w, w^{\prime}, \ldots, w^{(m)}\right)$ are marked as $W(P):=\max _{r \in I}\{p(r)\}$ and $\operatorname{deg}(P):=$ $\max _{r \in I}\left\{\operatorname{deg}\left(M_{r}\right)\right\}$, separately.

Considering the complex, ordinary differential equations

$$
P\left(w, w^{\prime}, \ldots, w^{(m)}\right)=b w^{n}+c .
$$

In (24), $b \neq 0, c$ are constants, and $n \in \mathbb{N}$.

We take $p, q \in \mathbb{N}$, and we regard the meromorphic solutions $w$ of (24) to have one or more poles. We can say that (24) is satisfied the $\langle p, q\rangle$ condition, where $p$ means that the equation has $p$ distinct meromorphic solutions and $q$ means that their multiplicity of the pole at $z=0$ is $q$.

It is difficult for us to find the $\langle p, q\rangle$ condition of (24), so we need a method to find the weak $\langle p, q\rangle$ condition showed as follows.

To find out the weak $\langle p, q\rangle$ condition of (24), we need to substitute Laurent series

$$
w(z)=\sum_{k=-q}^{\infty} c_{k} z^{k}, \quad q>0, c_{-q} \neq 0
$$

into (24); then we can find out the p distinct Laurent singular parts as below:

$$
\sum_{k=-q}^{-1} c_{k} z^{k}
$$

Given two complex numbers $\omega_{1}, \omega_{2}$, and $\operatorname{Im}\left(\omega_{1} / \omega_{2}\right)>0$, $L=L\left[2 \omega_{1}, 2 \omega_{2}\right]$ are discrete subset $L\left[2 \omega_{1}, 2 \omega_{2}\right]=\{\omega \mid \omega=$ $\left.2 n \omega_{1}+2 m \omega_{2}, n, m \in \mathbb{Z}\right\}$, which is isomorphic to $\mathbb{Z} \times \mathbb{Z}$. Let the discriminant $\Delta=\Delta\left(c_{1}, c_{2}\right):=c_{1}^{3}-27 c_{2}^{2}$ and

$$
s_{n}=s_{n}(L):=\sum_{\omega \in L \backslash\{0\}} \frac{1}{\omega^{n}} .
$$

A meromorphic function $w(z)$ means that $w(z)$ is holomorphic in the complex plane $\mathbb{C}$ except for poles. $\wp\left(z, g_{2}, g_{3}\right)$ is the Weierstrass elliptic function $[43,44]$ with invariants $g_{2}$ and $g_{3}$.

If $f$ is an elliptic function, or a rational function of $e^{\alpha z}, \alpha \in \mathbb{C}$, or a rational function of $z$, then we say that the meromorphic function $f$ belongs to the class $W$.

Weierstrass elliptic function $\wp(z):=\wp\left(z, g_{2}, g_{3}\right)$ is a meromorphic function with double periods $\omega_{1}, \omega_{2}$ and defined as

$$
\begin{aligned}
& \wp\left(z ; w_{1}, w_{2}\right):=\frac{1}{z^{2}}+\sum_{\mu, v \in \mathbf{Z}, \mu^{2}+\nu^{2} \neq 0}\left\{\frac{1}{\left(z+\mu w_{1}+\nu w_{2}\right)^{2}}\right. \\
& \left.-\frac{1}{\left(\mu w_{1}+\nu w_{2}\right)^{2}}\right\},
\end{aligned}
$$

which satisfies the following:

$$
\left(\wp^{\prime}(z)\right)^{2}=4 \wp(z)^{3}-g_{2} \wp(z)-g_{3},
$$

and in (29), $g_{2}=60 s_{4}, g_{3}=140 s_{6}$ and $\Delta\left(g_{2}, g_{3}\right) \neq 0$. 
Or alternating (29) to the form

$$
\left(\wp^{\prime}(z)\right)^{2}=4\left(\wp(z)-e_{1}\right)\left(\wp(z)-e_{2}\right)\left(\wp(z)-e_{2}\right),
$$

in $(30), e_{1}=\wp\left(\omega_{1}\right), e_{2}=\wp\left(\omega_{2}\right), e_{3}=\wp\left(\omega_{1}+\omega_{2}\right)$.

Contrarily, given two complex numbers $g_{2}$ and $g_{3}$ and $\Delta\left(g_{2}, g_{3}\right) \neq 0$, then there will have double periods $\omega_{1}, \omega_{2}$ Weierstrass elliptic function $\wp(z)$ which the solutions will possess.

In 2009, Eremenko [45] et al. investigated the $m$-order Briot-Bouquet equation (BBEq) as follows:

$$
F\left(w, w^{(m)}\right)=\sum_{j=0}^{m} F_{j}(w)\left(w^{(m)}\right)^{j}=0 .
$$

In (31), $F_{j}(w)$ are constant coefficients polynomials, $m \in$ $\mathbb{N}$. For the $m$ order BBEq, there are the following lemmas.

Lemma 4 (see [39-42]). Let $p, l, m, n \in \mathbb{N}$, and $\operatorname{deg} P(w$, $\left.w^{(m)}\right)<n$. Considering that a $m$-order Briot-Bouquet equation

$$
P\left(w^{(m)}, w\right)=b w^{n}+c
$$

satisfies weak $\langle p, q\rangle$ condition, then all the meromorphic solutions $w$ will belong to the class $W$. For some values of parameters, if the solution $w$ exists, then other meromorphic solutions will form a one-parametric family $w\left(z-z_{0}\right), z_{0} \in \mathbb{C}$. Furthermore, it can be written as the following forms of each elliptic solution with pole at $z=0$ :

$$
\begin{aligned}
& w(z) \\
& =\sum_{i=1}^{l-1} \sum_{j=2}^{q_{i}} \frac{(-1)^{j} c_{-i j}}{(j-1) !} \frac{d^{j-2}}{d z^{j-2}}\left(\frac{1}{4}\left[\frac{\wp^{\prime}(z)+B_{i}}{\wp(z)-A_{i}}\right]^{2}-\wp(z)\right) \\
& \quad+\sum_{i=1}^{l-1} \frac{c_{-i 1}}{2} \frac{\wp^{\prime}(z)+B_{i}}{\wp(z)-A_{i}}+\sum_{j=2}^{q_{l}} \frac{(-1)^{j} c_{-l j}}{(j-1) !} \frac{d^{j-2}}{d z^{j-2}} \wp(z) \\
& \quad+c_{0} .
\end{aligned}
$$

In (33), $c_{-i j}$ are given by equation (23), and $B_{i}^{2}=4 A_{i}^{3}-$ $g_{2} A_{i}-g_{3}, \sum_{i=1}^{l} c_{-i 1}=0$.

Each rational function solution $w:=R(z)$ can be show as the following form:

$$
R(z)=\sum_{i=1}^{l} \sum_{j=1}^{q} \frac{c_{i j}}{\left(z-z_{i}\right)^{j}}+c_{0},
$$

with $l(\leq p)$ distinct poles of multiplicity $q$.

Every simply periodic solution is a rational function $R(\xi)$ of $\xi=e^{\alpha z}(\alpha \in \mathbb{C}) . R(\xi)$ has $l(\leq p)$ distinct poles of multiplicity $q$ and can be show as the following form:

$$
R(\xi)=\sum_{i=1}^{l} \sum_{j=1}^{q} \frac{c_{i j}}{\left(\xi-\xi_{i}\right)^{j}}+c_{0} .
$$

Lemma 5 (see $[43,44])$. Weierstrass elliptic functions $\wp(z):=$ $\wp\left(z, g_{2}, g_{3}\right)$ have two successive degeneracies and addition formula:

(I) Degeneracy to simply periodic functions (i.e., rational functions of one exponential $e^{k z}$ ) according to

$$
\wp\left(z, 3 d^{2},-d^{3}\right)=2 d-\frac{3 d}{2} \operatorname{coth}^{2} \sqrt{\frac{3 d}{2}} z,
$$

if one root $e_{j}$ is double $\left(\Delta\left(g_{2}, g_{3}\right)=0\right)$.

(II) Degeneracy to rational functions of $z$ according to

$$
\wp(z, 0,0)=\frac{1}{z^{2}}
$$

if one root $e_{j}$ is triple $\left(g_{2}=g_{3}=0\right)$.

(III) Addition formula

$$
\begin{aligned}
\wp\left(z-z_{0}\right)= & -\wp(z)-\wp\left(z_{0}\right) \\
& +\frac{1}{4}\left[\frac{\wp^{\prime}(z)+\wp^{\prime}\left(z_{0}\right)}{\wp(z)-\wp\left(z_{0}\right)}\right]^{2} .
\end{aligned}
$$

By the above lemma and results, we introduce complex method to find exact solutions of some PDEs. The detailed five steps are as follows:

(1) Put the transform $T: u(x, t) \longrightarrow w(z),(x, t) \longrightarrow z$ into a given $P D E$ to produce a nonlinear ODE.

(2) Put (25) into (24) or (32) to find out the weak $\langle p, q\rangle$ condition.

(3) By determinant relation equation (33)-(35) we will, respectively, find the elliptic, rational and simply periodic solutions $u(z)$ of (24) or (32) with pole at $z=0$.

(4) By Lemmas 3 and 4, we obtain meromorphic solutions and the addition formula.

(5) Put the inverse transform $T^{-1}$ into these meromorphic solutions $w\left(z-z_{0}\right)$, all exact solutions $u(x, t)$ of the original PDE will be found.

More details of complex method can be found in [46-50].

2.2. Introduction of $\exp (-\phi(z))$-Expansion Method. Consider that a nonlinear partial differential equation (PDE) in the following form:

$$
P\left(\mu, \mu_{x}, \mu_{y}, \mu_{t}, \mu_{x x}, \mu_{y y}, \mu_{t t} \ldots\right)=0
$$

In (39), $\mathrm{P}$ is a polynomial with an unknown function $\mu(x, y, t)$ and its derivatives in which nonlinear terms and highest order derivatives are involved. And it can be processed as follows.

Step 1. Insert the traveling wave transform $\mu(x, y, t)=$ $w(z), z=k x+l y+r t$ into (39) alternating it to the following ordinary differential equation (ODE):

$$
K\left(w, w^{\prime}, w^{\prime \prime}, w^{\prime \prime \prime}, \ldots\right)=0
$$

and in (40), $K$ is a polynomial of $w(z)$ and its derivatives. 
Step 2. Regarding that (40) has the following traveling wave solution:

$$
w(z)=\sum_{j=0}^{n} C_{j}(\exp (-\phi(z)))^{j}
$$

in (41), $C_{j}(0 \leq j \leq n)$ are constants and will be determined later and $C_{j} \neq 0$ and $\phi=\phi(z)$ satisfies the ODE as follows:

$$
\phi^{\prime}(z)=\exp (-\phi(z))+\mu \exp (\phi(z))+\delta
$$

Equation (42) has different style solutions as follows: If $\delta^{2}-4 \mu>0, \mu \neq 0$, then

$$
\begin{aligned}
& \phi(z) \\
& =\ln \left(\frac{-\sqrt{\left(\delta^{2}-4 \mu\right)} \tanh \left(\left(\sqrt{\left(\delta^{2}-4 \mu\right)} / 2\right)(z+c)-\delta\right)}{2 \mu}\right),
\end{aligned}
$$

$\phi(z)$

$$
=\ln \left(\frac{-\sqrt{\left(\delta^{2}-4 \mu\right)} \operatorname{coth}\left(\left(\sqrt{\left(\delta^{2}-4 \mu\right)} / 2\right)(z+c)-\delta\right)}{2 \mu}\right) .
$$

$$
\text { If } \delta^{2}-4 \mu<0, \mu \neq 0 \text {, then }
$$

$\phi(z)$

$$
\begin{aligned}
& =\ln \left(\frac{-\sqrt{\left(4 \mu-\delta^{2}\right)} \tan \left(\left(\sqrt{\left(4 \mu-\delta^{2}\right)} / 2\right)(z+c)-\delta\right)}{2 \mu}\right), \\
& \phi(z) \\
& =\ln \left(\frac{-\sqrt{\left(4 \mu-\delta^{2}\right)} \cot \left(\left(\sqrt{\left(4 \mu-\delta^{2}\right)} / 2\right)(z+c)-\delta\right)}{2 \mu}\right) .
\end{aligned}
$$

$$
\text { If } \delta^{2}-4 \mu<0, \mu=0, \delta \neq 0 \text {, then }
$$

$$
\phi(z)=-\ln \left(\frac{\delta}{\exp (\delta(z+c)-1)}\right) .
$$

If $\delta^{2}-4 \mu=0, \mu \neq 0, \delta \neq 0$, then

$$
\phi(z)=\ln \left(-\frac{2(\delta(z+c)+2)}{\delta^{2}(z+c)}\right)
$$

If $\delta^{2}-4 \mu=0, \mu=0, \delta=0$, then

$$
\phi(z)=\ln (z+c) .
$$

In above equations, $C_{n} \neq 0, \delta$, and $\mu$ are constants and will be determined later and $c$ is an arbitrary constant. We consider the homogeneous balance between nonlinear terms and highest order derivatives of (40), so we can find the positive integer $n$.
Step 3. Putting (41) into (40) and accounting the function $\exp (-\phi(z))$, we get a polynomial of $\exp (-\phi(z))$. Calculating all the coefficients of the same power of $\exp (-\phi(z))$ to zero and then we get a set of algebraic equations. By solving the algebraic equations, we get the values of $C_{n} \neq 0, \delta$, and $\mu$, and then we put these into (34) along with(43)-(49) to get the determination of the solutions of (39).

\section{Proof of Theorems 1 and 2}

3.1. Proof of Theorem 1. Putting (25) into (2), we have $q=1$, $p=2, c_{-1}= \pm \sqrt{-6 \beta k^{2} / \tau}, c_{0}=0, c_{1}=\omega / k \tau c_{-1}, c_{2}=d /\left(\beta k^{3}-\right.$ $4 k \tau)$, and $c_{4}=-\omega d / 24 \beta^{2} k^{6}$ and $c_{3}$ is arbitrary constant.

Because (2) satisfies weak $\langle 2,1\rangle$ condition and is a twoorder $\mathrm{mKdv}$ equation, (2) satisfies the dominant condition. By Lemma 4, we know that all meromorphic solutions of (2) belong to $W$. Now we will give the forms of all meromorphic solutions of (2).

By (2), we infer the indeterminant rational solutions of (2) with pole at $z=0$ that

$$
u_{r}(z)=\frac{c_{11}}{z}+\frac{c_{12}}{z-z_{1}}+c_{10} .
$$

Putting $u_{r}(z)$ into (2), we get two classes: one is

$$
R_{1,1}(z)= \pm \sqrt{-\frac{6 \beta k^{2}}{\tau}} \frac{1}{z}
$$

in $(51), \omega=0, d=0$.

And the other one is

$$
R_{1,2}(z)= \pm \sqrt{-\frac{6\left(\beta k^{3}\right)}{k \tau z_{1}^{2}}}\left(\frac{z_{1}}{z}-\frac{z_{1}}{z-z_{1}}-1\right),
$$

in (52), $\omega=-6 \beta k^{3} / z_{1}^{2}, d=\mp(2 / 3) k \tau\left(-6 \beta k^{2} / \tau z_{1}^{2}\right)^{3 / 2}$. All rational solutions of (2) are as follows:

$$
w_{r, 1}(z)= \pm \sqrt{-\frac{6 \beta k^{2}}{\tau}} \frac{1}{z-z_{0}}
$$

and

$$
w_{r, 2}(z)= \pm \sqrt{-\frac{6 \beta k^{2}}{\tau z_{1}^{2}}}\left(\frac{z_{1}}{z-z_{0}}-\frac{z_{1}}{z-z_{0}-z_{1}}-1\right) .
$$

In (53)-(54), $z_{0} \in \mathbb{C}, \omega=0, d=0$, or in (54), $\omega=$ $-6 \beta k^{3} / z_{1}^{2}, d=\mp(2 / 3) k \tau\left(-6 \beta k^{2} / \tau z_{1}^{2}\right)^{3 / 2}$.

In order to get simply periodic solutions, we set $\xi=$ $\exp (\alpha z)$ and then put $u=u(\xi)$ into (2). We get

$$
\beta k^{3} \alpha^{2}\left(\xi R^{\prime}+\xi^{2} R^{\prime \prime}\right)-\omega R+\frac{k \tau}{3} R^{3}+d=0 .
$$

By putting

$$
u_{2}(\xi)=\frac{c_{2}}{\xi-1}+\frac{c_{1}}{\xi-\xi_{1}}+c_{20}
$$


into (55), we get

$$
\begin{aligned}
& R_{2,1}(\xi)=\frac{\alpha}{1-\xi_{1}} \\
& \quad \cdot \sqrt{-\frac{6 \beta k^{2}}{\tau}}\left(-2\left(\xi_{1}-1\right)\left(\frac{1}{\xi-1}-\frac{1}{\xi_{1}-1}\right)\right. \\
& \left.\quad+\left(\xi_{1}+1\right)\right),
\end{aligned}
$$

in (57), $\omega=-\left(1 / 2+6 \xi_{1} /\left(1-\xi_{1}\right)^{2}\right) \beta k^{3} \alpha^{2}, d=\left(8 k \tau \xi_{1}\left(\xi_{1}+\right.\right.$ 1) $\left.\alpha^{3} / 3\left(1-\xi_{1}\right)^{3}\right)\left(-6 \beta k^{2} / \tau z_{1}^{2}\right)^{3 / 2}$.

And the other solution is

$$
R_{2,2}(\xi)=\alpha \sqrt{-\frac{6 \beta k^{2}}{\tau}}\left(\frac{1}{\xi-1}+1\right),
$$

in (58), $\omega=-\beta k^{3} \alpha^{2} / 2, d=0$.

So for $z=0$, all simply periodic solutions of (2) are gotten, which are

$$
\begin{aligned}
& w_{s 0,1}(z)=\alpha \sqrt{-\frac{6 \beta k^{2}}{\tau}}\left(\operatorname{coth} \frac{\alpha}{2} z-\operatorname{coth} \frac{\alpha}{2}\left(z-z_{1}\right)\right. \\
& \left.-\operatorname{coth} \frac{\alpha}{2} z_{1}\right)
\end{aligned}
$$

and

$$
w_{s 0,2}(z)=\alpha \sqrt{-\frac{6 \beta k^{2}}{\tau}} \tanh \frac{\alpha}{2} z .
$$

So all simply periodic solutions of (2) are gotten:

$$
\begin{gathered}
w_{s, 1}(z)=\alpha \sqrt{-\frac{6 \beta k^{2}}{\tau}}\left(\operatorname{coth} \frac{\alpha}{2}\left(z-z_{0}\right)\right. \\
\left.-\operatorname{coth} \frac{\alpha}{2}\left(z-z_{0}-z_{1}\right)-\operatorname{coth} \frac{\alpha}{2} z_{1}\right),
\end{gathered}
$$

in $(61), z_{0} \in, \omega=-\beta k^{3} \alpha^{2}\left(1 / 2+\left(3 / 2 \sinh ^{2}(\alpha / 2) z_{1}\right)\right), d=$ $\sqrt{-6 \beta k^{2} / \tau}\left(\tanh (\alpha / 2) z_{1} / \sinh ^{2}(\alpha / 2) z_{1}\right)$, and $z_{1} \neq 0$.

And the other solution is

$$
w_{s, 2}(z)=\alpha \sqrt{-\frac{6 \beta k^{2}}{\tau}} \tanh \frac{\alpha}{2}\left(z-z_{0}\right)
$$

in (62), $\omega=-\beta k^{3} \alpha^{2} / 2, d=0$.

By (33) of Lemma 4, we have indeterminant relations of elliptic solutions of (2) with the pole at $z=0$

$$
u_{d 0}(z)=\frac{c_{-1}}{2} \frac{\wp^{\prime}(z)+F}{\wp(z)-E}+c_{30}
$$

in (63), $F^{2}=4 E^{3}-g_{2} E-g_{3}$. Applying the conclusion II of Lemma 5 to $u_{d 0}(z)$ and noting that the results of rational solutions obtained above, we deduce that $c_{30}=0, E=F=0$, and $g_{3}=0$. Then we get that

$$
u_{d 0}(z)= \pm \frac{1}{2} \sqrt{-\frac{6 \beta k^{2}}{\tau} \frac{\wp^{\prime}(z)}{\wp(z)}},
$$

in (64), $g_{3}=0$.

So all elliptic function solutions of (2) are

$$
u_{d 0}(z)= \pm \frac{1}{2} \sqrt{-\frac{6 \beta k^{2}}{\tau}} \frac{\wp^{\prime}\left(z-z_{0}\right)}{\wp\left(z-z_{0}\right)},
$$

$$
w_{d}(z)= \pm \frac{1}{2} \sqrt{-\frac{6 \beta k^{2}}{\tau}} \frac{(-\wp+c)\left(4 \wp c^{2}+4 \wp^{2} c+2 \wp^{\prime} a-\wp g_{2}-c g_{2}\right)}{\left(\left(12 c^{2}-g_{2}\right) \wp+4 c^{3}-3 c g_{2}\right) \wp^{\prime}+\left(4 \wp^{3}+12 c \wp^{2}-3 g_{2} \wp-c g_{2}\right) d^{\prime}}
$$

In (66), $g_{3}=0, a^{2}=4 c^{3}-g_{2} c$, and $g_{2}$ and $c$ are arbitrary constants.

3.2. Proof of Theorem 2. Taking the homogeneous balance between $u^{\prime \prime}$ and $u^{3}$ in (2) we get

$$
u(z)=C_{0}+C_{1} \exp (-\phi(z))
$$

in (67), $C_{1} \neq 0$ and $C_{0}$ are constants which need to be determined, and $\phi(z)$ satisfies equation $\phi^{\prime}(z)=\exp (-\phi(z))+$ $\mu \exp (\phi(z))+\delta$, whereas $\delta$ and $\mu$ are arbitrary constants.
From (67), we insert $u, u^{3}, u^{\prime \prime}$ into (2) and sort out the coefficients of $\exp (-\phi(z))$ to zero, and then we obtain

$$
\begin{aligned}
& c_{1}= \pm \sqrt{\frac{-6 \beta k^{2}}{\tau}} \\
& c_{0}= \pm \frac{1}{2} \lambda \sqrt{\frac{-6 \beta k^{2}}{\tau}}
\end{aligned}
$$

in (68), $\lambda= \pm \sqrt{2\left(2 \beta k^{3} \mu-\omega\right) / \beta k^{3}}$ and $d=0$. 
By putting (68) into (67), we get

$$
u_{1}(z)=\frac{1}{2} \lambda \sqrt{\frac{-6 \beta k^{2}}{\tau}}+\sqrt{\frac{-6 \beta k^{2}}{\tau}} \exp (-\phi(z)),
$$

or

$$
u_{2}(z)=-\frac{1}{2} \lambda \sqrt{\frac{-6 \beta k^{2}}{\tau}}-\sqrt{\frac{-6 \beta k^{2}}{\tau}} \exp (-\phi(z)) .
$$

We apply (43)-(49) into (69) and (70), respectively, to obtain traveling wave solutions of the $\mathrm{mKdV}$ equation as follows.

$$
\begin{aligned}
& \text { If } \delta^{2}-4 \mu>0, \mu \neq 0 \text {, } \\
& u_{11}(z)=\frac{1}{2} \lambda \sqrt{\frac{-6 \beta k^{2}}{\tau}}+\sqrt{\frac{-6 \beta k^{2}}{\tau}} \\
& \cdot \frac{2 \mu}{\sqrt{\left(\delta^{2}-4 \mu\right)} \tanh \left(\left(\sqrt{\delta^{2}-4 \mu} / 2\right)(z+c)+\delta\right)} \text {, } \\
& u_{12}(z)=\frac{1}{2} \lambda \sqrt{\frac{-6 \beta k^{2}}{\tau}}+\sqrt{\frac{-6 \beta k^{2}}{\tau}} \\
& \cdot \frac{2 \mu}{\sqrt{\left(\delta^{2}-4 \mu\right)} \operatorname{coth}\left(\left(\sqrt{\delta^{2}-4 \mu} / 2\right)(z+c)+\delta\right)} \text {, } \\
& u_{21}(z)=-\frac{1}{2} \lambda \sqrt{\frac{-6 \beta k^{2}}{\tau}}-\sqrt{\frac{-6 \beta k^{2}}{\tau}} \\
& \cdot \frac{2 \mu}{\sqrt{\left(\delta^{2}-4 \mu\right)} \tanh \left(\left(\sqrt{\delta^{2}-4 \mu} / 2\right)(z+c)+\delta\right)} \text {, } \\
& u_{22}(z)-\frac{1}{2} \lambda \sqrt{\frac{-6 \beta k^{2}}{\tau}}-\sqrt{\frac{-6 \beta k^{2}}{\tau}} \\
& \cdot \frac{2 \mu}{\sqrt{\left(\delta^{2}-4 \mu\right)} \operatorname{coth}\left(\left(\sqrt{\delta^{2}-4 \mu} / 2\right)(z+c)+\delta\right)} \text {. }
\end{aligned}
$$

If $\delta^{2}-4 \mu<0, \mu \neq 0$,

$$
\begin{gathered}
u_{13}(z)=\frac{1}{2} \lambda \sqrt{\frac{-6 \beta k^{2}}{\tau}}+\sqrt{\frac{-6 \beta k^{2}}{\tau}} \\
\cdot \frac{2 \mu}{\sqrt{\left(\delta^{2}-4 \mu\right)} \tanh \left(\left(\sqrt{\delta^{2}-4 \mu} / 2\right)(z+c)+\delta\right)} \\
u_{14}(z)=\frac{1}{2} \lambda \sqrt{\frac{-6 \beta k^{2}}{\tau}}+\sqrt{\frac{-6 \beta k^{2}}{\tau}} \\
\cdot \frac{2 \mu}{\sqrt{\left(\delta^{2}-4 \mu\right)} \operatorname{coth}\left(\left(\sqrt{\delta^{2}-4 \mu / 2}\right)(z+c)+\delta\right)},
\end{gathered}
$$

$$
\begin{aligned}
& u_{23}(z)=-\frac{1}{2} \lambda \sqrt{\frac{-6 \beta k^{2}}{\tau}}-\sqrt{\frac{-6 \beta k^{2}}{\tau}} \\
& \frac{2 \mu}{\sqrt{\left(\delta^{2}-4 \mu\right)} \tanh \left(\left(\sqrt{\delta^{2}-4 \mu} / 2\right)(z+c)+\delta\right)} \\
& u_{24}(z)=-\frac{1}{2} \lambda \sqrt{\frac{-6 \beta k^{2}}{\tau}}-\sqrt{\frac{-6 \beta k^{2}}{\tau}} \\
& \cdot \frac{2 \mu}{\sqrt{\left(\delta^{2}-4 \mu\right)} \operatorname{coth}\left(\left(\sqrt{\delta^{2}-4 \mu} / 2\right)(z+c)+\delta\right)} \text {. } \\
& \text { If } \delta^{2}-4 \mu>0, \mu=0, \delta \neq 0, \\
& u_{15}(z)=\frac{1}{2} \lambda \sqrt{\frac{-6 \beta k^{2}}{\tau}}+\sqrt{\frac{-6 \beta k^{2}}{\tau}} \frac{\delta}{\exp (\delta(z+c))-1}, \\
& u_{25}(z)=-\frac{1}{2} \lambda \sqrt{\frac{-6 \beta k^{2}}{\tau}} \\
& -\sqrt{\frac{-6 \beta k^{2}}{\tau}} \frac{\delta}{\exp (\delta(z+c))-1} . \\
& \text { If } \delta^{2}-4 \mu=0, \mu \neq 0, \delta \neq 0 \text {, } \\
& u_{16}(z)=\frac{1}{2} \lambda \sqrt{\frac{-6 \beta k^{2}}{\tau}}+\sqrt{\frac{-6 \beta k^{2}}{\tau}} \frac{\delta^{2}(z+c)}{2(\delta(z+c)+2)}, \\
& u_{26}(z)=-\frac{1}{2} \lambda \sqrt{\frac{-6 \beta k^{2}}{\tau}}-\sqrt{\frac{-6 \beta k^{2}}{\tau}} \frac{\delta^{2}(z+c)}{2(\delta(z+c)+2)} \text {. } \\
& \text { If } \delta^{2}-4 \mu=0, \mu=0, \delta=0 \text {, } \\
& u_{17}(z)=\frac{1}{2} \lambda \sqrt{\frac{-6 \beta k^{2}}{\tau}}+\sqrt{\frac{-6 \beta k^{2}}{\tau}} \frac{1}{z+c}, \\
& u_{27}(z)=-\frac{1}{2} \lambda \sqrt{\frac{-6 \beta k^{2}}{\tau}}-\sqrt{\frac{-6 \beta k^{2}}{\tau}} \frac{1}{z+c} \text {. }
\end{aligned}
$$

\section{Computer Simulations}

In this section, we will show some computer simulation pictures to illustrate some results. Considering $k=1, l=1$, $r=1$, the simply periodic solutions are shown in Figure 1, and the rational function solutions $w_{r, 1}$ are shown in Figure 2. And through the $\exp (-\phi(z))$-expansion method, we get some other simply periodic solutions. We take the solutions $u_{s, 11}(z)$ to further analyze their properties by Figure 3.

4.1. The Physical Significance of the Figures. Figures 1, 2, and 3 shows Waveform Graphs of several functions at different times. We can see that there will be some distinct generation poles shown in Figures. 

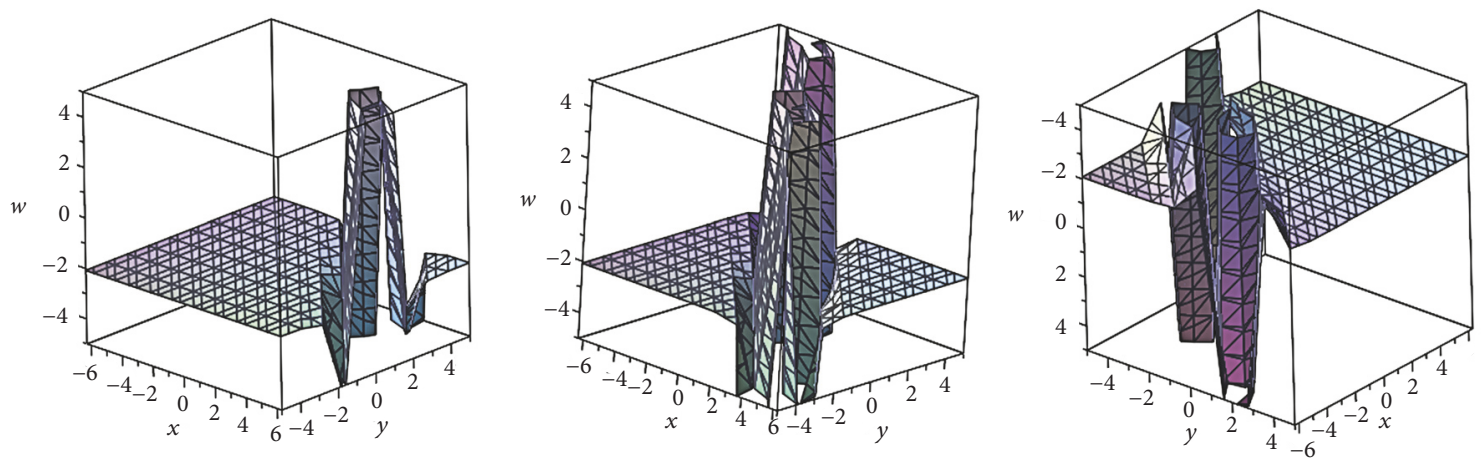

FIGURE 1: The solution of $\mathrm{mKdV}$ equation corresponding to $w_{s, 1}$, take $\alpha=2, \sqrt{-6 \beta k^{2} / \tau}=1$, and $z_{0}=1, z_{1}=2$; from left to right, $t$ take the following three different values: (a) $t=-5$, (b) $t=0$, and (c) $t=5$.
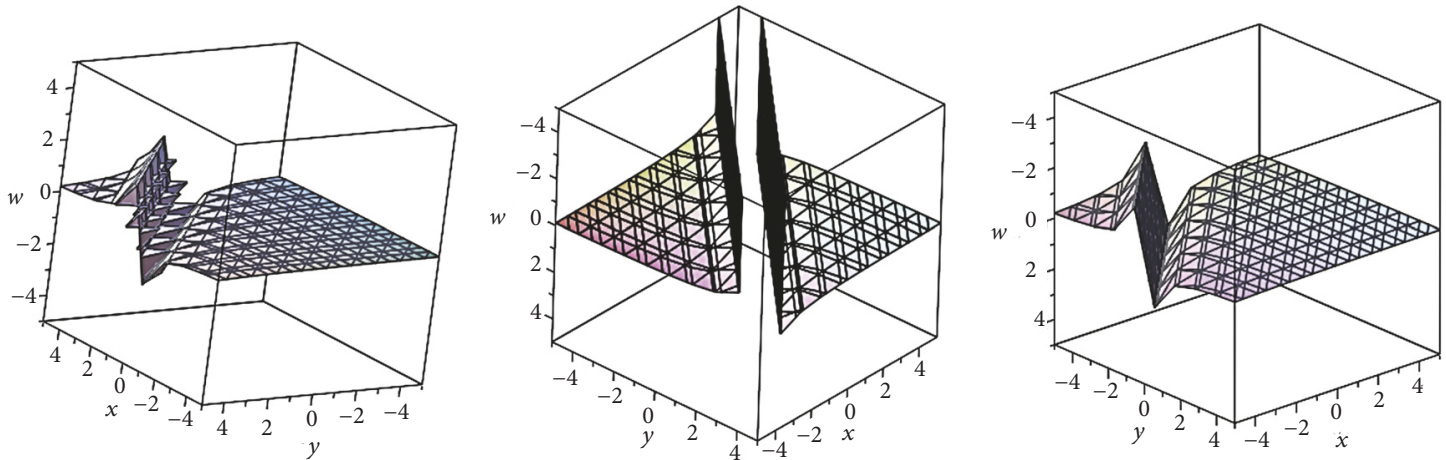

FIgURE 2: The solution of $\mathrm{mKdV}$ equation corresponding to $w_{r, 1}$, take $6 \beta k^{2} / \tau=1$; from left to right, $t$ take the following three different values: (a) $t=-5$, (b) $t=0$, and (c) $t=5$.
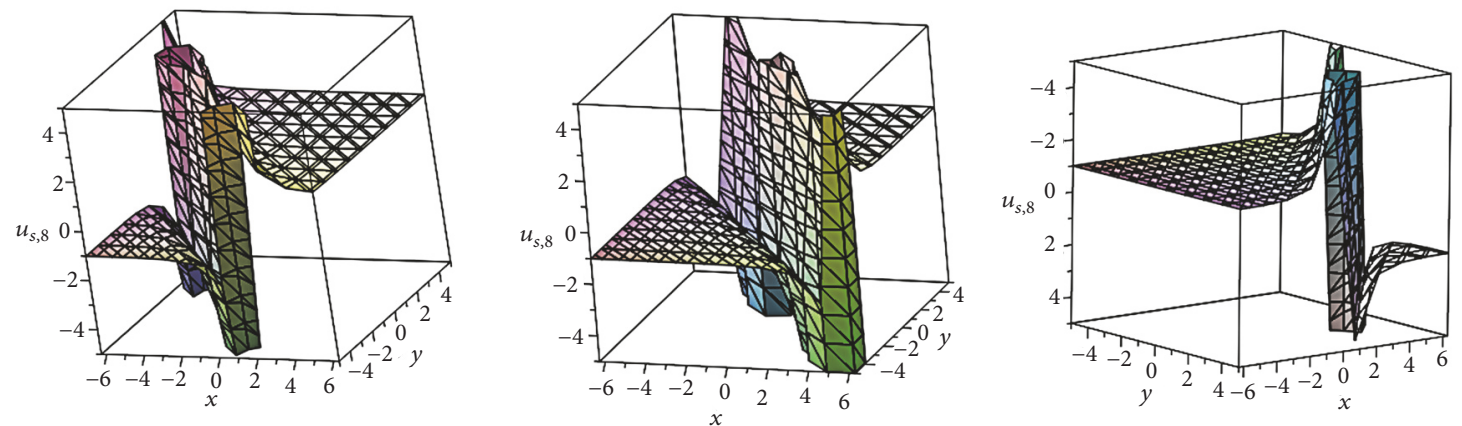

FIgURE 3: The solution of $\mathrm{mKdV}$ equation corresponding to $u_{s, 11}$, take $\lambda=2, \beta k^{2} / \tau=-1 / 6, \mu=2$, and $\delta^{2}-\mu=2$; from left to right, $t$ take the following three different values: (a) $t=-5$, (b) $t=0$, and (c) $t=5$.

\section{Conclusions}

It can be seen from the above analysis that the complex method and $\exp (-\phi(z))$-expansion method are powerful tools for solving the exact solutions of nonlinear evolution equations. The general meromorphic solutions of $(2+1)$ dimensional $\mathrm{mKdV}$ equation are obtained by the complex method, and we found eight solutions of $(2+1)$-dimensional $\mathrm{mKdV}$ equation. Using $\exp (-\phi(z))$-expansion method, we also find fourteen solutions of $(2+1)$-dimensional $\mathrm{mKdV}$ equation. By comparing with the two methods, we find more solutions by $\exp (-\phi(z))$-expansion method, while we can say that the solutions of the elliptic function are only obtained by the complex method.

\section{Data Availability}

No data were used to support this study. 


\section{Conflicts of Interest}

The authors declare that they have no conflicts of interest.

\section{Authors' Contributions}

All authors typed, read, and approved the final manuscript.

\section{Acknowledgments}

The work presented in this paper is supported by the Plateau Disciplines in Shanghai. Also this work was supported by Leading Academic Applied Mathematical of Shanghai Dianji University (16JCXK02) and Humanity and Social Science Youth foundation of Ministry of Education (18YJC630120).

\section{References}

[1] L. Gabora, "Nonlinear science and the psyche," Foundations of Science, vol. 5, no. 3, pp. 391-393, 2000.

[2] T. Bartsch and J. a. Mederski, "Nonlinear time-harmonic Maxwell equations in domains," Journal of Fixed Point Theory and Applications, vol. 19, no. 1, pp. 959-986, 2017.

[3] A. Heuer, C. F. E. Schroer, D. Diddens, C. Rehwald, and M. Blank-Burian, "Nonlinear response from the perspective of energy landscapes and beyond," The European Physical Journal Special Topics, vol. 226, no. 14, pp. 3061-3078, 2017.

[4] G. Damien, "Inverse scattering at fixed energy for radial magnetic schrodinger operators with obstacle in dimension two," Annales Henri Poincare', vol. 19, no. 10, pp. 3089-3128, 2018.

[5] C.-Q. Dai, D.-S. Wang, L.-L. Wang, J.-F. Zhang, and W. M. Liu, "Quasi-two-dimensional Bose-Einstein condensates with spatially modulated cubic-quintic nonlinearities," Annals of Physics, vol. 326, no. 9, pp. 2356-2368, 2011.

[6] D. Ding, D. Jin, and C. Dai, "Analytical solutions of DifferentialDifference Sine-Gordon equation," Thermal Science, vol. 21, pp. 1701-1705, 2017.

[7] L.-Q. Kong, J. Liu, D.-Q. Jin, D.-J. Ding, and C.-Q. Dai, "Soliton dynamics in the three-spine $\alpha$-helical protein with inhomogeneous effect," Nonlinear Dynamics, vol. 87, no. 1, pp. 83-92, 2017.

[8] A. R. Aguirre, T. R. Araujo, J. F. Gomes, and A. H. Zimerman, "Type-II Backlund transformations via gauge transformations," Journal of High Energy Physics, vol. 2011, no. 12, p. 56, 2011.

[9] X. Liu, H. Triki, Q. Zhou et al., "Generation and control of multiple solitons under the influence of parameters," Nonlinear Dynamics, vol. 95, no. 1, pp. 143-150, 2019.

[10] C. Yang, W. Liu, Q. Zhou, D. Mihalache, and B. A. Malomed, "One-soliton shaping and two-soliton interaction in the fifthorder variable-coefficient nonlinear Schrödinger equation," Nonlinear Dynamics, vol. 95, no. 1, pp. 369-380, 2019.

[11] W. Liu, Y. Zhang, H. Triki et al., "Interaction properties of solitonics in inhomogeneous optical fibers," Nonlinear Dynamics, vol. 95, no. 1, pp. 557-563, 2019.

[12] B. F. Samsonov and A. A. Pecheritsyn, "The Darboux transform for the one-dimensional stationary dirac equation," Russian Physics Journal, vol. 43, no. 11, pp. 938-943, 2000.

[13] X. Hao, H. Wang, L. Liu, and Y. Cui, "Positive solutions for a system of nonlinear fractional nonlocal boundary value problems with parameters and p-Laplacian operator," Boundary Value Problems, vol. 2017, no. 182, 2017.
[14] J. Jiang, L. Liu, and Y. Wu, "Symmetric positive solutions to singular system with multi-point coupled boundary conditions," Applied Mathematics and Computation, vol. 220, pp. 536-548, 2013.

[15] Y. Wang and J. Jiang, "Existence and nonexistence of positive solutions for the fractional coupled system involving generalized p-laplacian," Advances in Difference Equations, vol. 2017, article 337, 2017.

[16] M. Zuo, X. Hao, L. Liu, and Y. Cui, "Existence results for impulsive fractional integro-differential equation of mixed type with constant coefficient and antiperiodic boundary conditions," Boundary Value Problems, vol. 2017, no. 161, 2017.

[17] A. Mao and H. Chang, "Kirchhoff type problems in RN with radial potentials and locally Lipschitz functional," Applied Mathematics Letters, vol. 62, pp. 49-54, 2016.

[18] A. Mao and W. Wang, "Nontrivial solutions of nonlocal fourth order elliptic equation of Kirchhoff type in R3," Journal of Mathematical Analysis and Applications, vol. 1, pp. 556-563, 2018.

[19] M. Shao and A. Mao, "Multiplicity of solutions to SchrödingerPoisson system with concave-convex nonlinearities," Applied Mathematics Letters, vol. 83, pp. 212-218, 2018.

[20] Y. Wang and Z. Zhao, "Existence and multiplicity of solutions for a second-order impulsive differential equation via variational methods," Advances in Difference Equations, vol. 2017, no. 46, 2017.

[21] L. Liu, F. Sun, X. Zhang, and Y. Wu, "Bifurcation analysis for a singular differential system with two parameters via to topological degree theory," Nonlinear Analysis: Modelling and Control, vol. 22, no. 1, pp. 31-50, 2017.

[22] F. Sun, L. Liu, X. Zhang, and Y. Wu, "Spectral analysis for a singular differential system with integral boundary conditions," Mediterranean Journal of Mathematics, vol. 13, no. 6, pp. 47634782, 2016.

[23] Y. Wang, L. Liu, X. Zhang, and Y. Wu, "Positive solutions of an abstract fractional semipositone differential system model for bioprocesses of HIV infection," Applied Mathematics and Computation, vol. 258, pp. 312-324, 2015.

[24] X. G. Zhang, L. S. Liu, Y. H. Wu, and B. Wiwatanapataphee, “The spectral analysis for a singular fractional differential equation with a signed measure," Applied Mathematics and Computation, vol. 257, pp. 252-263, 2015.

[25] M. Wang, Y. Zhou, and Z. Li, "Application of a homogeneous balance method to exact solutions of nonlinear equations in mathematical physics," Physics Letters A, vol. 216, no. 1-5, pp. 67-75, 1996.

[26] W. Malfliet and W. Hereman, "The tanh method. I. Exact solutions of nonlinear evolution and wave equations," Physica Scripta, vol. 54, no. 6, pp. 563-568, 1996.

[27] M. A. Abdou, "The extended tanh method and its applications for solving nonlinear physical models," Applied Mathematics and Computation, vol. 190, no. 1, pp. 988-996, 2007.

[28] D. Lü, "Jacobi elliptic function solutions for two variant Boussinesq equations," Chaos, Solitons \& Fractals, vol. 24, no. 5, pp. 1373-1385, 2005.

[29] Z. Yan, "Abundant families of Jacobi elliptic function solutions of the (2+1)-dimensional integrable Davey-Stewartson-type equation via a new method," Chaos, Solitons \& Fractals, vol. 18, no. 2, pp. 299-309, 2003.

[30] M. Wang and X. Li, "Extended F-expansion method and periodic wave solutions for the generalized Zakharov equations," Physics Letters A, vol. 343, no. 1, pp. 48-54, 2005. 
[31] M. Wang and X. Li, "Applications of F-expansion to periodic wave solutions for a new Hamiltonian amplitude equation," Chaos, Solitons \& Fractals, vol. 24, no. 5, pp. 1257-1268, 2005.

[32] K. Khan and M. A. Akbar, "The $\exp (-\phi(z))$-expansion method for finding travelling wave solutions of Vakhnenko-Parkes equation," International Journal of Dynamical Systems and Differential Equations, vol. 5, no. 1, p. 72, 2014.

[33] S. M. R. Islam, K. Khan, and M. A. Akbar, "Exact solutions of unsteady Korteweg-de Vries and time regularized long wave equations," SpringerPlus, vol. 4, no. 1, p. 124, 2015.

[34] Y. Gu and J. Qi, "Symmetry reduction and exact solutions of two higher-dimensional nonlinear evolution equations," Journal of Inequalities and Applications, vol. 2017, no. 1, article 314, 2017.

[35] M. A. Alejo, "Geometric breathers of the mKdV equation," Acta Applicandae Mathematicae, vol. 121, no. 1, pp. 137-155, 2012.

[36] Y. Huang, Y. Wu, F. Meng, and W. Yuan, "All exact traveling wave solutions of the combined KDV-mKDV Equation," Advances in Difference Equations, vol. 2014, no. 1, article 261, 2014.

[37] S. Singh and S. Saha Ray, "Exact solutions for the Wick-type stochastic Kersten-Krasil'shchik coupled KdV-mKdV equations," The European Physical Journal Plus, vol. 132, no. 11, p. 480, 2017.

[38] J.-q. Mei and H.-q. Zhang, "New soliton-like and periodiclike solutions for the KdV equation," Applied Mathematics and Computation, vol. 169, no. 1, pp. 589-599, 2005.

[39] W. Yuan, Y. Huang, and Y. Shang, "All traveling wave exact solutions of two nonlinear physical models," Applied Mathematics and Computation, vol. 219, no. 11, pp. 6212-6223, 2013.

[40] W. Yuan, B. Xiao, Y. Wu, and J. Qi, "The general traveling wave solutions of the Fisher type equations and some related problems," Journal of Inequalities and Applications, vol. 2014, no. 1, article 500, 2014

[41] W. Yuan, Z. Huang, M. Fu, and J. Lai, “The general solutions of an auxiliary ordinary differential equation using complex method and its applications," Advances in Difference Equations, vol. 2014, pp. 1-9, 2014.

[42] W. Yuan, F. Meng, Y. Huang, and Y. Wu, "All traveling wave exact solutions of the variant Boussinesq equations," Applied Mathematics and Computation, vol. 268, pp. 865-872, 2015.

[43] S. Lang, "Elliptic functions," Graduate Texts in Mathematics, vol. 112, no. 1, pp. 71-86, 1987.

[44] R. Conte and M. Musette, "Elliptic general analytic solutions," Studies in Applied Mathematics, vol. 123, no. 1, pp. 63-81, 2009.

[45] A. E. Eremenko, L. Liao, and T. W. Ng, "Meromorphic solutions of higher order Briot-Bouquet differential equations," Mathematical Proceedings of the Cambridge Philosophical Society, vol. 146, no. 1, pp. 197-206, 2009.

[46] Y. Y. Gu, N. Aminakbari, W. J. Yuan, and Y. H. Wu, "Meromorphic solutions of a class of algebraic differential equations related to Painlevé equation III," House Journal of Mathematics, vol. 43, no. 4, pp. 1045-1055, 2017.

[47] Y. Gu, W. Yuan, N. Aminakbari, and Q. Jiang, "Exact solutions of the Vakhnenko-Parkes equation with complex method," Journal of Function Spaces, vol. 2017, Article ID 6521357, 6 pages, 2017.

[48] Y. Gu, W. Yuan, N. Aminakbari, and J. Lin, "Meromorphic solutions of some algebraic differential equations related Painlevé equation IV and its applications," Mathematical Methods in the Applied Sciences, vol. 41, no. 10, pp. 3832-3840, 2018.

[49] Y. Gu, Deng B., and J. Lin, "Exact traveling wave solutions to the (2+1)-dimensional Jaulent-Miodek equation," Advances in Mathematical Physics, vol. 2018, Article ID 5971646, 9 pages, 2018.
[50] Y. Gu, X. Zheng, and F. Meng, "Painlevé analysis and abundant meromorphic solutions of a class of nonlinear algebraic differential equations," Mathematical Problems in Engineering, vol. 2019, Article ID 9210725, 11 pages, 2019. 


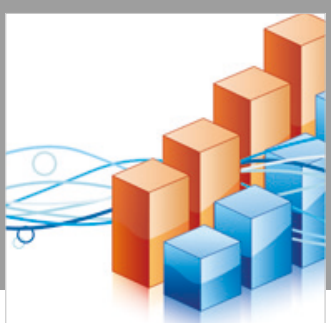

Advances in

Operations Research

\section{-n-m}
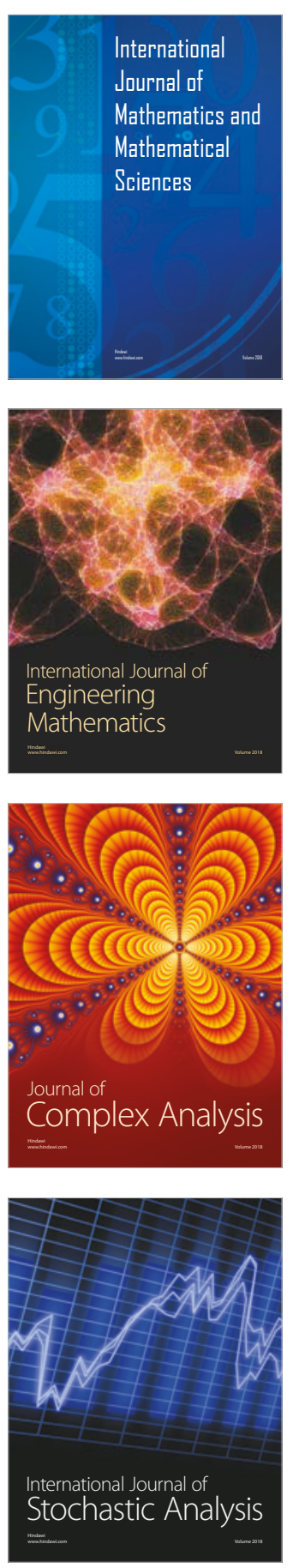
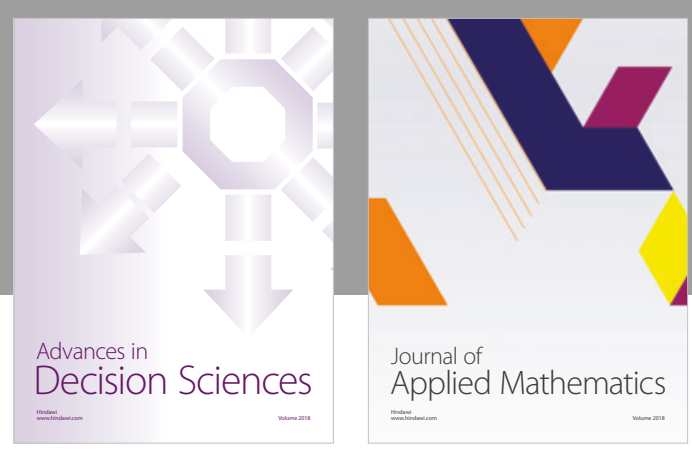

Journal of

Applied Mathematics
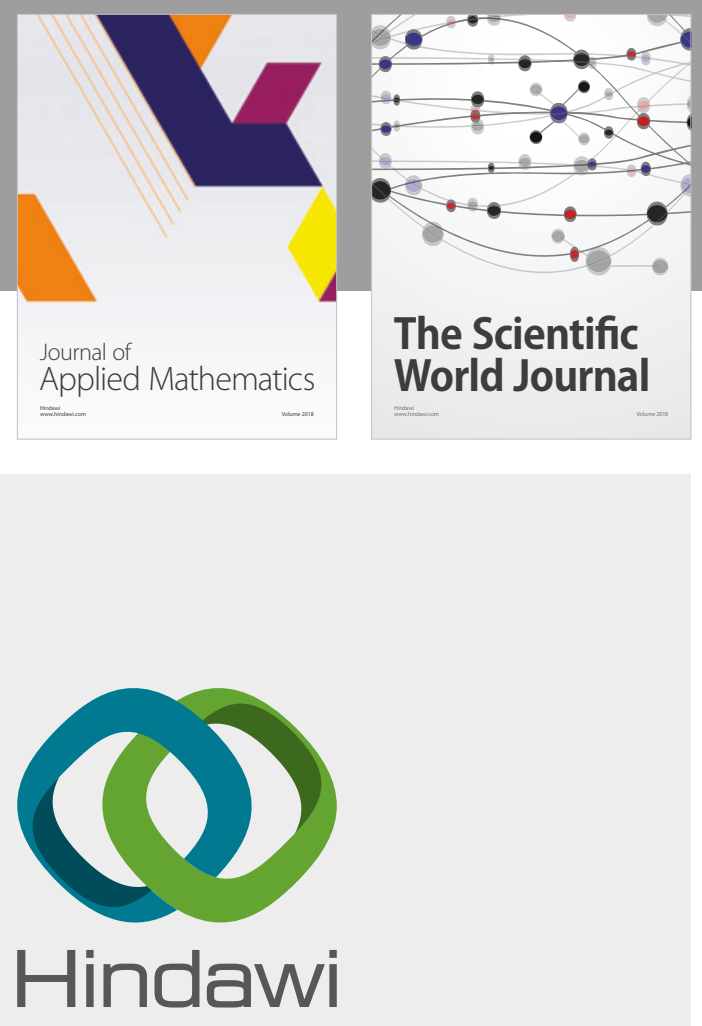

Submit your manuscripts at

www.hindawi.com

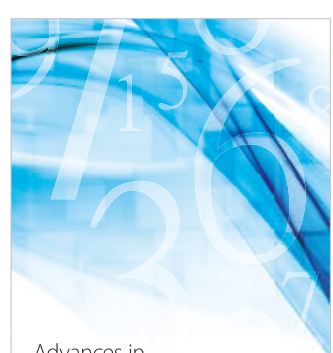

Advances in
Numerical Analysis
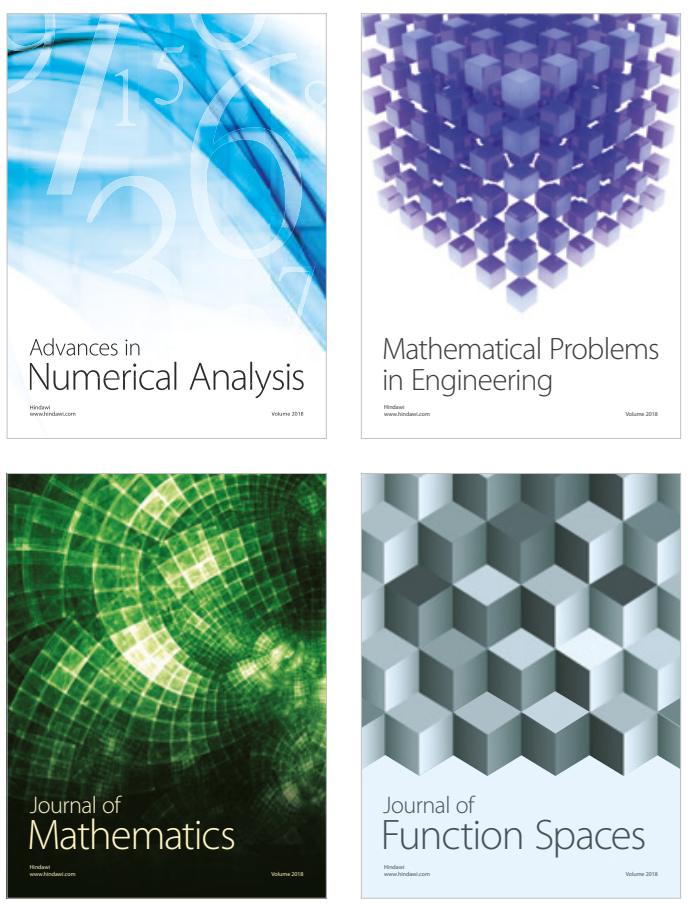

Mathematical Problems in Engineering

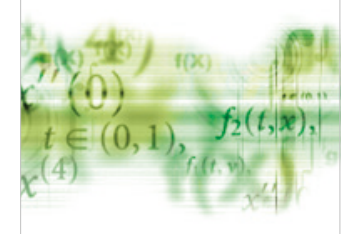

International Journal of

Differential Equations

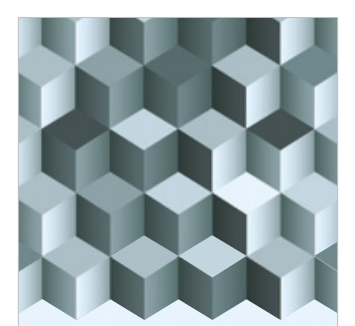

Journal of

Function Spaces

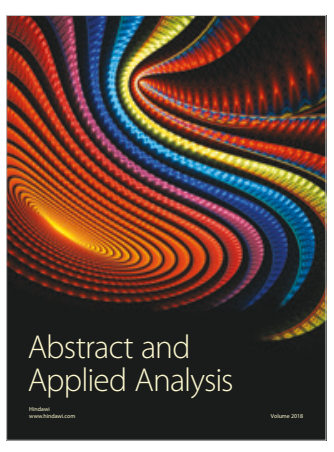

The Scientific

World Journal

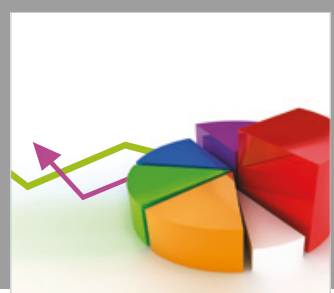

Journal of

Probability and Statistics
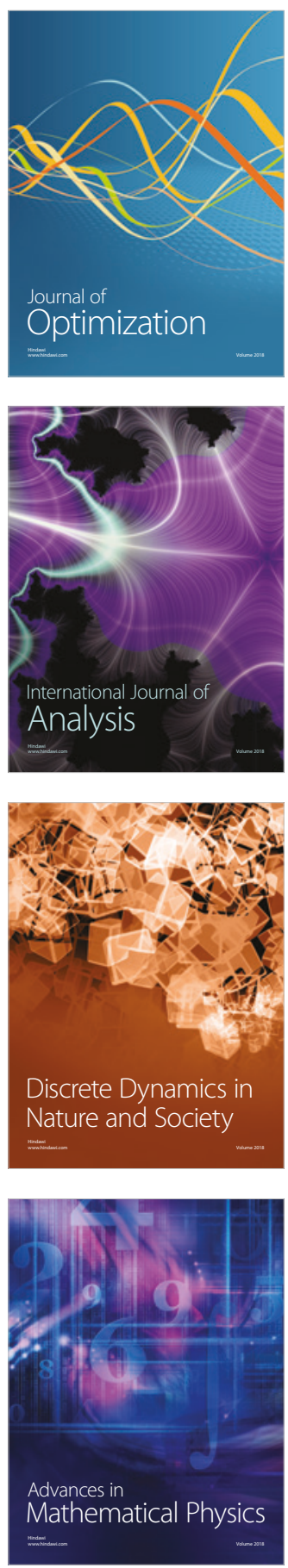